УДК 519.21

\title{
Variational Analysis of a Dynamic Electroviscoelastic Problem with Friction
}

\author{
Aziza Bachmar* \\ Souraya Boutechebak ${ }^{\dagger}$ \\ Touffik Serrar \\ Department of Mathematics, Faculty of Sciences \\ Ferhat Abbas University of Setif-1, 19000
}

Algeria

Received 06.04.2018, received in revised form 06.07.2018, accepted 06.08.2018

A dynamic contact problem is considered in the paper. The material behavior is described by electrovisco-elastic constitutive law with piezoelectric effects. The body is in contact with a rigide obstacle. Contact is described with the Signorini condition, a version of Coulomb's law of dry friction, and with a regularized electrical conductivity condition. A variational formulation of the problem is derived. Under the assumption that coefficient of friction is small, existence and uniqueness of a weak solution of the problem is proved. The proof is based on evolutionary variational inequalities and fixed points of operators.

Keywords: piezoelectric, frictional contact, visco-elastic, fixed point, dynamic process, coulomb's law of friction, variational inequality.

DOI: 10.17516/1997-1397-2019-12-1-68-78.

\section{Introduction}

The piezoelectric effect is the accumulation of electric charges on surfaces of particular crystals after deformation. The reverse effect consists of the generation of stress and strain in crystals under the action of the electric field on the boundary. A deformable material with such a behaviour is called a piezoelectric material. Piezoelectric materials are used extensively as switches and in many engineering systems. Various models have been developed to describe the interaction between electric and mechanical fields (see, e.g. [9-11]). Therefore there is a need to extend the results on models of contact with deformable bodies which include coupling between mechanical and electrical properties. General models for elastic materials with piezoelectric effects can be found in [9-11] and more recently in [2], for viscoelastic piezoelectric materials in [3, 13, 14]. Elasto-viscoplastic piezoelectric materials have been also studied [7].

In this paper, we consider a general model for the dynamic process of frictional contact between a deformable body and a rigid obstacle. The material obeys an electro-viscoelastic constitutive law with piezoelectric effects. Moreover, to describe the contact and friction the Signorini condition and a non local Coulomb's law of friction are used. We derive a variational formulation of the problem which includes a variational second order evolution inequality. We establish the existence of a unique weak solution of the problem. The idea is to reduce the second order evolution inequality of the system to first order evolution inequality. After this, we use classical results on first order evolution inequalities and equations and the fixed point arguments.

The paper is structured as follows. In Section 1 we present the electro visco-elastic contact model with friction and provide comments on the contact boundary conditions. In Section 2

\footnotetext{
*Aziza_bechmar@yahoo.fr

†bou_souraya @yahoo.fr

${ }_{\ddagger}^{\ddagger}$ serrartouffik@yahoo.fr

(c) Siberian Federal University. All rights reserved
} 
we list the assumptions on the data and derive the variational formulation. In Section 3 we present our main results on existence and uniqueness which state the unique weak solvability of the Signorini contact electro-visco-elastic problem with non local Coulomb's law of friction conditions.

\section{Problem statement}

Let us consider a body made of a piezoelectric material which occupies the domain $\Omega \subset \mathbb{R}^{d}$ $(d \leqslant 3)$ with a lipschitz boundary $\Gamma$. The body is described by the electro-visco-elastic constituve law with piezoelectric effects. Let $[0 . T]$ be the time interval where $T>0$, let $\Gamma$ be split into three parts $\Gamma_{1}, \Gamma_{2}$ and $\Gamma_{3}$ such that meas $\Gamma_{1}>0$. We assume that the body is fixed on $\Gamma_{1}$ and surface tractions of density $h$ act on $\Gamma_{2}$. The body may come into contact with a rigide obstacle on $\Gamma_{3}$. In the other hand, $\Gamma$ is split into two measurable sets $\Gamma_{a}$ and $\Gamma_{b}$ such that meas $\Gamma_{b}>0$ and $\Gamma_{3} \subset \Gamma_{b}$. We assume that the electrical potential $q_{0}$ acts on $\Gamma_{a}$, and surface electric charge of density $q_{2}$ acts on $\Gamma_{b}$. We assume that the problem is quasistatic. The piezoelectric effect is the accumulation of electric charges on surfaces of particular crystals after deformation. The space of second order symmetric tensors in the space $\mathbb{R}^{d}$ is denoted by $\mathbb{S}^{d}$. Character - means inner product in the space $\mathbb{R}^{d}$,and the Euclidean norm in the space $\mathbb{S}^{d}$ is denoted by $|$.$| . The$ unit outward normal to $\Gamma$ is denoted by $\nu$. The classical formulation of the electro-visco-elastic contact friction problem is as follows.

Problem P : Find displacement field $u: \Omega \times[0 . T] \rightarrow \mathbb{R}^{d}$, stress field $\sigma: \Omega \times[0 . T] \rightarrow \mathbb{S}^{d}$, electric potential field $\varphi: \Omega \times[0 . T] \rightarrow \mathbb{R}$ and electric displacement field $D: \Omega \times[0 . T] \rightarrow \mathbb{R}^{d}$ such that:

$$
\begin{aligned}
& \sigma=\mathcal{A} \varepsilon(\dot{u})+\mathcal{G} \varepsilon(u)-\xi^{*} E(\varphi) \text { in } \Omega \times[0 . T], \\
& D=\beta E(\varphi)+\xi \varepsilon(u) \text { in } \Omega \times[0 . T] \text {, } \\
& \rho \ddot{u}=D i v \sigma+f_{0} \text { in } \Omega \times[0 . T] \text {, } \\
& \operatorname{div} D=q_{0} \quad \text { in } \Omega \times[0 . T] \text {, } \\
& u=0 \quad \text { on } \Gamma_{1} \times[0 . T] \text {, } \\
& \sigma_{\nu}=h \quad \text { on } \Gamma_{2} \times[0 . T], \\
& u_{\nu} \leqslant 0, \sigma_{\nu} \leqslant 0, u_{\nu} \sigma_{\nu}=0 \quad \text { on } \Gamma_{3} \times[0 . T], \\
& \left\{\begin{array}{l}
\left|\sigma_{\tau}\right| \leqslant \mu p\left|R \sigma_{\nu}\right|\left|\sigma_{\tau}\right|<\mu p\left|R \sigma_{\nu}\right| \Longrightarrow \dot{u}_{\tau}=0 \\
\left|\sigma_{\tau}\right|=\mu p\left|R \sigma_{\nu}\right| \Longrightarrow \exists \lambda \geqslant 0 \text { such that } \sigma_{\tau}=-\lambda \dot{u}_{\tau}
\end{array} \quad \text { on } \Gamma_{3} \times[0 . T],\right. \\
& \varphi=0 \quad \text { on } \Gamma_{a} \times[0 . T], \\
& D \nu=q_{2} \quad \text { on } \Gamma_{b} \times[0 . T], \\
& u(0)=u_{0} \quad \text { and } \dot{u}(0)=v_{0} \quad \text { in } \Omega \times[0 . T] \text {. }
\end{aligned}
$$

Where (1.1) and (1.2) are the electro-visco-elastic constitutive law of the material, $\varepsilon(u)$ is the linearized strain tensor, $E(\varphi)=-\nabla \varphi$ is the electric field, $\mathcal{A}$ is the viscosity nonlinear tensor, $\mathcal{G}$ is the elasticity tensor, $\xi, \xi^{*}$ are the third order piezoelectric tensor and its transpose, $\beta$ is the electric permittivity tensor. Equation (1.3) is the equation of motion, where $\rho$ represents the mass density. Equation (1.4) is the equilibrium equation. Div $\sigma$, and $\operatorname{div} D$ are divergence operators. Relations (1.5) and (1.6) are displacement and traction boundary conditions. Relations (1.7) and (1.8) describe the Signorini contact with non local Coulomb's law of friction conditions, where $u$ ${ }_{\nu}$ and $u_{\tau}$ are the normal and tangential displacements, $\sigma_{\nu}$ and $\sigma_{\tau}$ ) are the normal and tangential stresses, $R$ is the normal regularization operator that is a linear and continuous operator $R$ : $H^{-\frac{1}{2}}(\Gamma) \rightarrow \mathbb{L}^{2}(\Gamma)$. 
Let us introduce a non-negative function $p$ the so-called friction bound and the coefficient of friction $\mu \geqslant 0$. The friction law used in some studies is $p(r)=r_{+}$, where $r_{+}=\max \{0, r\}$. Recently, a new version of Coulomb's law was proposed from thermodynamic considerations. It has the form

$$
p(r)=r(1-\alpha r)_{+},
$$

where $\alpha$ is a small positive coefficient related to the hardness and the wear of the contact surface. Relations (1.9) and (1.10) represent the electric boundary conditions. Finally, in (1.11) $u_{0}$ is the initial displacement, and $v_{0}$ is the initial velocity.

\section{Variational formulation and preliminaries}

For a weak formulation of the problem we itroduce some notation. The indices $i, j, k, l$ range from 1 to $d$ and summation over repeated indices is implied. An index that follows a comma represents the partial derivative with respect to the corresponding spatial variable, e. $\mathrm{g}, u_{i . j}=$ $=\frac{\partial u_{i}}{\partial x_{j}}$. We also use the following notations:

$$
\begin{aligned}
& H=\mathbb{L}^{2}(\Omega)^{d}=\left\{u=\left(u_{i}\right) / u_{i} \in \mathbb{L}^{2}(\Omega)\right\}, \\
& \mathcal{H}=\left\{\sigma=\left(\sigma_{i j}\right) / \sigma_{i j}=\sigma_{j i} \in \mathbb{L}^{2}(\Omega)\right\} \\
& H_{1}=\left\{u=\left(u_{i}\right) / \varepsilon(u) \in \mathcal{H}\right\}, \\
& \mathcal{H}_{1}=\{\sigma \in \mathcal{H} / \operatorname{Div} \sigma \in H\} .
\end{aligned}
$$

The operators of deformation $\varepsilon$ and divergence Div are defined as follows

$$
\varepsilon(u)=\left(\varepsilon_{i j}(u)\right), \varepsilon_{i j}(u)=\frac{1}{2}\left(u_{i, j}+u_{j, i}\right), \operatorname{Div} \sigma=\left(\sigma_{i j, j}\right) .
$$

The spaces $H, \mathcal{H}, H_{1}$, and $\mathcal{H}_{1}$ are real Hilbert spaces with the canonical inner products defined as follows

$$
\begin{aligned}
(u, v)_{H} & =\int_{\Omega} u_{i} v_{i} d x, \forall u, v \in H, \\
(\sigma, \tau)_{\mathcal{H}} & =\int_{\Omega} \sigma_{i j} \tau_{i j} d x, \forall \sigma, \tau \in \mathcal{H}, \\
(u, v)_{H_{1}} & =(u, v)_{H}+(\varepsilon(u), \varepsilon(v))_{\mathcal{H}}, \forall u, v \in H_{1}, \\
(\sigma, \tau)_{\mathcal{H}_{1}} & =(\sigma, \tau)_{\mathcal{H}}+(\operatorname{Div} \sigma, \operatorname{Div} \tau)_{H}, \sigma, \tau \in \mathcal{H}_{1} .
\end{aligned}
$$

The associated norm in the space $H\left(\mathcal{H}, H_{1}\right.$, and $\mathcal{H}_{1}$, respectively) is denoted by $|\cdot|_{H}\left(|\cdot|_{\mathcal{H}},|\cdot|_{H_{1}}\right.$, and $|\cdot|_{\mathcal{H} 1}$, respectively).

We use standard notation for $\mathbb{L}^{p}$ and Sobolev spaces associated with $\Omega$ and $\Gamma$. For a function $\psi \in H^{1}(\Omega)$ we still write $\psi$ to denote its trace on $\Gamma$. We recall that the summation convention applies to a repeated index.

For the electric displacement field we use two Hilbert spaces:

$$
\mathcal{W}=\mathbb{L}^{2}(\Omega)^{d}, \quad \mathcal{W}_{1}=\left\{D \in \mathcal{W}, \operatorname{div} D \in \mathbb{L}^{2}(\Omega)\right\}
$$

with the inner products

$$
(D, E)_{\mathcal{W}}=\int_{\Omega} D_{i} E_{i} d x, \quad(D, E)_{\mathcal{W}_{1}}=(D, E)_{\mathcal{W}}+(\operatorname{div} D, \operatorname{div} E)_{\mathbb{L}^{2}(\Omega)}
$$

and associated norms $|\cdot|_{\mathcal{W}}$ and $|\cdot|_{\mathcal{W}_{1}}$ ). The electric potential field is to be found in:

$$
W=\left\{\psi \in H^{1}(\Omega), \psi=0 \text { on } \Gamma_{a}\right\} .
$$


Since meas $\left(\Gamma_{a}\right)>0$, the following Friedrichs-Poincaré' inequality

$$
|\nabla \psi|_{\mathcal{W}} \geqslant c_{F}|\psi|_{H^{1}(\Omega)} \quad \forall \psi \in W
$$

holds, where $c_{F}>0$ is a constant which depends only on $\Omega$ and $\Gamma_{a}$. We use on $W$ the inner product

$$
(\varphi, \psi)_{W}=(\nabla \varphi, \nabla \psi)_{\mathcal{W}}
$$

and the associated norm $|\cdot|_{W}$. It follows from $(2.1)$ that $|\cdot|_{H^{1}(\Omega)}$ and $|\cdot|_{W}$ are equivalent norms in $W$. Therefore $\left(W,|\cdot|_{W}\right)$ is the real Hilbert space.

Moreover, it follows from the Sobolev trace theorem that there exists a constant $\widetilde{c}_{0}$ depending only on $\Omega, \Gamma_{a}$ and $\Gamma_{3}$ such that

$$
|\psi|_{L^{2}\left(\Gamma_{3}\right)} \leqslant \widetilde{c}_{0}|\psi|_{W} \quad \forall \psi \in W .
$$

We recall that when $D \in \mathcal{W}_{1}$ is a sufficiently regular function the Green type formula

$$
(D, \nabla \psi)_{\mathcal{W}}+(\operatorname{div} D, \psi)_{\mathbb{L}^{2}(\Omega)}=\int_{\Gamma} D \nu \cdot \psi d a
$$

holds. When $\sigma$ is a regular function, the following Green type formula

$$
(\sigma, \varepsilon(v))_{\mathcal{H}}+(D i v \sigma, v)_{H}=\int_{\Gamma} \sigma \nu \cdot v d a \quad \forall v \in H_{1}
$$

holds. Next, we define the space

$$
V=\left\{u \in H_{1} / u=0 \text { on } \Gamma_{1}\right\} .
$$

Since meas $\left(\Gamma_{1}\right)>0$, the following Korn inequality

$$
|\varepsilon(u)|_{\mathcal{H}} \geqslant c_{K}|v|_{H_{1}} \quad \forall v \in V
$$

holds, where $c_{K}>0$ is a constant which depends only on $\Omega$ and $\Gamma_{1}$. We use in the space $V$ the inner product

$$
(u, v)_{V}=(\varepsilon(u), \varepsilon(v))_{\mathcal{H}},
$$

and the associated norm $|\cdot|_{V}$. It follows from (2.5) that the norms $|\cdot|_{H_{1}}$ and $|\cdot|_{V}$ are equivalent norms on $V$, and therefore $\left(V,|\cdot|_{V}\right)$ is a real Hilbert space. Moreover, it follows from the Sobolev trace Theorem that there exists a constant $c_{0}$ depending only on domain $\Omega, \Gamma_{1}$ and $\Gamma_{3}$ such that

$$
|v|_{L^{2}\left(\Gamma_{3}\right)^{d}} \leqslant c_{0}|v|_{V} \quad \forall v \in V .
$$

Finally, for a real Banach space $\left(X,|\cdot|_{X}\right)$ we use the usual notation for the space $\mathbb{L}^{p}(0 . T ; X)$ and $W^{k \cdot p}(0 . T ; X)$, where $1 \leqslant p \leqslant \infty, k=1,2, \ldots$ The spaces of continuous and continuously differentiable function on $[0 . T]$ with values in $X$ are denoted by $C(0 . T ; X)$ and $C^{1}(0 . T ; X)$. The corresponding norms are

$$
\begin{aligned}
& |x|_{C(0 . T ; X)}=\max _{t \in[0 . T]}|x(t)|_{X}, \\
& |x|_{C^{1}(0 . T ; X)}=\max _{t \in[0 . T]}|x(t)|_{X}+\max _{t \in[0 . T]}|\dot{x}(t)|_{X} .
\end{aligned}
$$


In what follows we assume the following assumptions for the problem $P$.

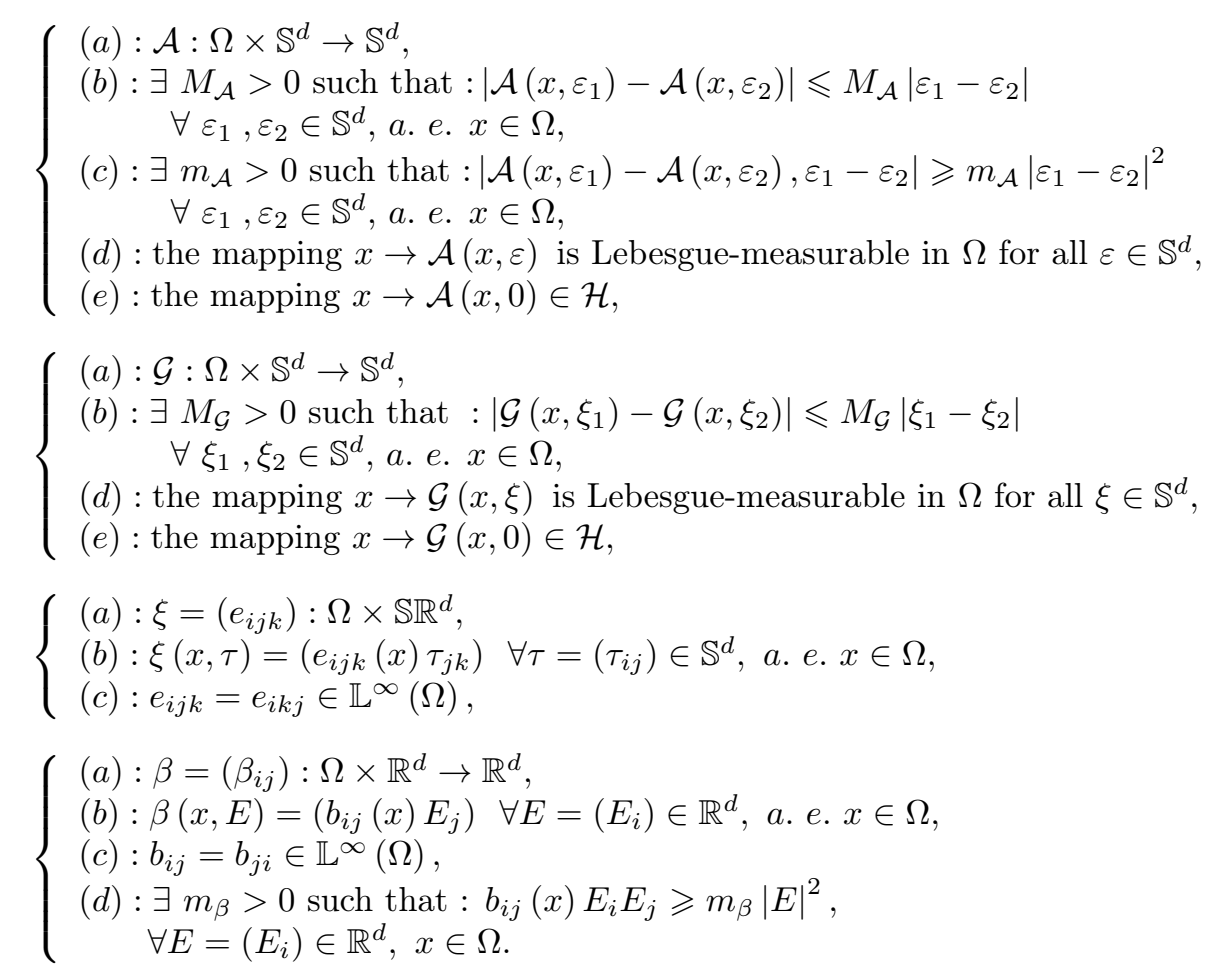

We deduce from assumptions (2.9) and (2.10) that the piezoelectric operator $\xi$ (the electric permittivity operator $\beta$ ) is linear,and it has measurable bounded component denoted by $e_{i j k}$ $\left(b_{i j}\right)$. Moreover, $\beta$ is a symmetric and positive definite operator.

One should also note that the transposed operator $\xi^{*}=\left(e_{i j k}^{*}\right)$, where $e_{i j k}^{*}=e_{k i j}$ and the following equality

$$
\xi \sigma . v=\sigma \cdot \xi^{*} v \quad \forall \sigma \in \mathbb{S}^{d}, \quad v \in \mathbb{R}^{d}
$$

holds. The friction function satisfies

$$
\left\{\begin{aligned}
& p: \Gamma_{3} \times \mathbb{R} \rightarrow \mathbb{R}_{+} \text {verifies: } \\
&(a): \exists M>0 \text { such that }:\left|p\left(x, r_{1}\right)-p\left(x, r_{2}\right)\right| \leqslant M\left|r_{1}-r_{2}\right| \\
& \quad \text { or every } r_{1}, r_{2} \in \mathbb{R}, \text { a.e. } x \in \Gamma_{3}, \\
&(b): \text { the mapping }: x \rightarrow p(x, r) \text { is measurable on } \Gamma_{3}, \text { for every } r \in \mathbb{R}, \\
&(c): p(x, 0)=0, \text { a. e. } x \in \Gamma_{3} .
\end{aligned}\right.
$$

We note that (2.11) is satisfied in the case when $p$ is given by (1.12).

We also assume that body forces and surface tractions satisfy the following requirements

$$
f_{0} \in \mathbb{L}^{2}(0 . T ; H), \quad h \in \mathbb{L}^{2}\left(0 . T ; \mathbb{L}^{2}\left(\Gamma_{2}\right)^{d}\right),
$$

as well as the densities of electric charges satisfy the following requirements

$$
q_{0} \in \mathbb{L}^{2}\left(0 . T ; \mathbb{L}^{2}(\Omega)\right), \quad q_{2} \in \mathbb{L}^{2}\left(0 . T ; \mathbb{L}^{2}\left(\Gamma_{b}\right)\right) .
$$

Function $f:[0 . T] \rightarrow V$ and $q:[0 . T] \rightarrow W$ is defined as

$$
\begin{aligned}
& (f(t), v)_{V}=\int_{\Omega} f_{0}(t) v d x+\int_{\Gamma_{2}} h(t) v d a \quad \forall v \in V, \quad t \in[0 . T] \\
& (q(t), \psi)_{W}=-\int_{\Omega} q_{0}(t) \psi d x+\int_{\Gamma_{b}} q_{2}(t) \psi d a \quad \forall \psi \in W, \quad t \in[0 . T]
\end{aligned}
$$


for all $u, v \in V, \psi \in W$ and $t \in[0 . T]$. We also note that conditions (2.12) and (2.13) imply

$$
f \in \mathbb{L}^{2}\left(0 . T ; V^{\prime}\right), \quad q \in \mathbb{L}^{2}(0 . T ; W),
$$

while the friction coefficient $\mu$, the mass density $\rho$ satisfy

$$
\begin{aligned}
& \mu \in \mathbb{L}^{\infty}\left(\Gamma_{3}\right), \mu(x) \geqslant 0, \text { a. e. on } \Gamma_{3}, \\
& \rho \in \mathbb{L}^{\infty}(\Omega) \text { there exists } \rho^{*}>0 \text { such that } \rho(x) \geqslant \rho^{*}, \text { a.e. } x \in \Omega, \\
& u_{0} \in V .
\end{aligned}
$$

The friction functional $j: \mathcal{H} \times V \rightarrow \mathbb{R}$ is denoted by

$$
j(\sigma, v)=\int_{\Gamma_{3}} \mu p\left|R \sigma_{\nu}\right|\left|v_{\tau}\right| d a .
$$

The convex subset of admissible displacements fields $U$ is given by

$$
U=\left\{v \in H_{1} / v=0 \text { on } \Gamma_{1}, v_{\nu} \leqslant 0 \text { on } \Gamma_{3}\right\}
$$

Using the standard procedure based on Green's formula, we obtain the following formulation of mechanical problem (1.3)-(1.10).

Problem PV: Find a displacement field $u: \Omega \times[0 . T] \rightarrow \mathbb{R}^{d}$, a stress field $\sigma: \Omega \times[0, T] \rightarrow \mathbb{S}^{d}$, an electric potential field $\varphi: \Omega \times[0, T] \rightarrow \mathbb{R}$, and an electric displacement field $D: \Omega \times[0, T] \rightarrow \mathbb{R}^{d}$ such that

$$
\begin{gathered}
(\ddot{u}, w-\dot{u})_{V^{\prime} \times V}+(\sigma(t), \varepsilon(w-\dot{u}(t)))_{\mathcal{H}}+j(\sigma, w)-j(\sigma, \dot{u}(t)) \geqslant(f(t), w-\dot{u}(t)) \forall u, w \in V, \\
(D(t), \nabla \psi)_{\mathbb{L}^{2}(\Omega)^{d}}+(q(t), \psi)_{W}=0 \quad \forall \psi \in W \\
u(0)=u_{0} \text { and } \dot{u}(0)=v_{0} .
\end{gathered}
$$

\section{Existence and uniqueness}

Our main result which states the unique solvability of the Problem is the following.

Theorem 1. Let assumptions (2.7)-(2.20) hold. Then, Problem PV has a unique solution $\{u, \sigma, \varphi, D\}$ which satisfies

$$
\begin{aligned}
& u \in C^{1}(0, H) \cap W^{1.2}(0, T V) \cap W^{2.2}\left(T ;^{\prime}\right), \\
& \varphi \in W^{1.2}(0, T W), \\
& \sigma \in \mathbb{L}^{2}(0, T \mathcal{H}), D i v \sigma \in \mathbb{L}^{2}\left(0 . T ; V^{\prime}\right), \\
& D \in W^{1.2}\left(0, T \mathcal{W}_{1}\right) .
\end{aligned}
$$

We conclude that under assumptions (2.7)-(2.20) mechanical problem (1.1)-(1.11) has a unique weak solution that satisfies (3.1)-(3.4). The proof of this theorem is carried out in several steps. It is based on the first order nonlinear evolutionary inequalities (see [4,6-8]), evolution equations (see Ref. [1]), and fixed point arguments.

Let us assume that $G \in \mathbb{L}^{2}(0 . T ; \mathcal{H})$ and $\eta \in \mathbb{L}^{2}\left(0 . T ; V^{\prime}\right)$ are given and obtain a variational formulation of Problem $P V$. 
Problem $\mathbf{P V}_{\mathbf{G} \eta}$ : Find a displacement field $u_{G \eta}:[0, T] \rightarrow V$ such that

$$
\begin{gathered}
u_{G \eta}(t) \in U, \quad\left(\ddot{u}_{G \eta}, w-\dot{u}_{G \eta}\right)_{V^{\prime} \times V}+\left(\mathcal{A} \varepsilon\left(\dot{u}_{G \eta}(t)\right), \varepsilon\left(w-\dot{u}_{G \eta}(t)\right)_{\mathcal{H}}+\right. \\
+\left(\eta, w-\dot{u}_{G \eta}(t)\right)_{V^{\prime} \times V}+j(G, w)-j\left(G, \dot{u}_{G \eta}(t)\right) \geqslant\left(f(t), w-\dot{u}_{G \eta}(t)\right) \forall w \in V, \\
\dot{u}_{G \eta}(0)=v(0)=v_{0} .
\end{gathered}
$$

We define $f_{\eta}(t) \in V$ for a.e.t $\in[0 . T]$ by

$$
\left(f_{\eta}(t), w\right)_{V^{\prime} \times V}=(f(t)-\eta(t), w)_{V^{\prime} \times V} \forall w \in V .
$$

It follows from (2.16) that:

$$
f_{\eta} \in \mathbb{L}^{2}\left(0 . T ; V^{\prime}\right) .
$$

Let now $u_{G \eta}:[0 . T] \rightarrow V$ be the function defined by

$$
u_{G \eta}(t)=\int_{0}^{t} v_{G \eta}(s) d s+u_{0} \quad \forall t \in[0 . T] .
$$

We define the operator $A: V^{\prime} \rightarrow V$ as

$$
(A v, w)_{V^{\prime} \times V}=(\mathcal{A} \varepsilon(v), \varepsilon(w))_{\mathcal{H}} \forall v, w \in V .
$$

Lemma 1. For all $G \in \mathbb{L}^{2}(0 . T ; \mathcal{H})$ and $\eta \in \mathbb{L}^{2}\left(0 . T ; V^{\prime}\right) P V_{G \eta}$ has a unique solution and

$$
v_{G \eta} \in C(0 . T ; H) \cap \mathbb{L}^{2}(0 . T ; V) \text { and } \dot{v}_{G \eta} \in \mathbb{L}^{2}\left(0 . T ; V^{\prime}\right) .
$$

Proof. The proof based on the first order nonlinear evolutionary inequalities is given in $([6,15])$.

Next we use the displacement field $u_{G \eta}$ and consider the following variational problem.

Problem $P V 1_{G \eta}$ : Find an electric potential field $\varphi_{G \eta}: \Omega \times[0 . T] \rightarrow W$ such that

$$
\left(\beta \nabla \varphi_{G \eta}(t), \nabla \psi\right)_{\mathbb{L}^{2}(\Omega)^{d}}-\left(\xi \varepsilon\left(u_{G \eta}(t)\right), \nabla \psi\right)_{\mathbb{L}^{2}(\Omega)^{d}}=(q(t), \psi)_{W} \quad \forall \psi \in W, t \in[0 . T] .
$$

We have the following result for $P V 1_{G \eta}$.

Lemma 2. There exists a unique solution $\varphi_{G \eta} \in W^{1.2}(0, T ; W)$ that satisfies (3.12). Moreover, if $\varphi_{1}$ and $\varphi_{2}$ are two solutions to (3.12) then there exists a constant $c>0$ sach that

$$
\left|\varphi_{1}(t)-\varphi_{2}(t)\right|_{W} \leqslant c\left|u_{1}(t)-u_{2}(t)\right|_{V} \forall t \in[0 . T] .
$$

Proof. Let $t \in[0, T]$ and we use the Riesz-Frechet representation theorem to define the operator $A_{G \eta}: W \rightarrow W$

$$
\left(A_{G \eta}(t) \varphi, \psi\right)_{W}=\left(\beta \nabla \varphi_{G \eta}(t), \nabla \psi\right)_{\mathbb{L}^{2}(\Omega)^{d}}-\left(\xi \varepsilon\left(u_{G \eta}(t)\right), \nabla \psi\right)_{\mathbb{L}^{2}(\Omega)^{d}} \forall t \in[0 . T] .
$$

The operator $A_{G \eta}(t)$ is a strongly monotone, Lipschits continuous operator on $W$ and, therefore, there exists a unique element $\varphi_{G \eta}(t) \in W$ such that

$$
A_{G \eta} \varphi_{G \eta}(t)=q(t) .
$$

We combine (3.14) and (3.15), and find that $\varphi_{G \eta}(t) \in W$ is the unique solution of nonlinear variational equation (3.12). We show that $\varphi_{\xi \eta} \in W^{1.2}(0, T W)$. Let us assume that $\eta_{1}, \eta_{2} \in$ $\mathbb{L}^{2}\left(0, T V^{\prime}\right), G_{1}, G_{2} \in \mathbb{L}^{2}(0, T \mathcal{H})$ and $\varphi_{G \eta}\left(t_{i}\right)=\varphi_{i}, u_{G \eta}\left(t_{i}\right)=u_{i}$. Using (3.12), we obtain

$$
m_{\beta}\left|\varphi_{1}-\varphi_{2}\right|_{W} \leqslant c_{\xi}\left|u_{1}-u_{2}\right|_{V} .
$$


We consider the operator

$\Lambda: \mathbb{L}^{2}\left(0 . T ; \mathcal{H} \times V^{\prime}\right) \rightarrow \mathbb{L}^{2}\left(0 . T ; \mathcal{H} \times V^{\prime}\right)$ defined as

$\Lambda(G, \eta)=\left(\Lambda_{1}(G), \Lambda_{2}(\eta)\right), \forall G \in \mathbb{L}^{2}(0 . T ; \mathcal{H}), \forall \eta \in \mathbb{L}^{2}\left(0 . T ; V^{\prime}\right)$,

$\left|\Lambda\left(G_{2}, \eta_{2}\right)-\Lambda\left(G_{1}, \eta_{2}\right)\right|^{2}=\left|\left(\Lambda_{1}\left(G_{2}\right), \Lambda_{2}\left(\eta_{2}\right)\right)-\left(\Lambda_{1}\left(G_{1}\right), \Lambda_{2}\left(\eta_{1}\right)\right)\right|^{2}$,

$\left|\Lambda_{1}\left(G_{2}\right)-\Lambda_{1}\left(G_{1}\right), \Lambda_{2}\left(\eta_{2}\right)-\Lambda_{2}\left(\eta_{1}\right)\right|^{2}=\left|\Lambda_{1}\left(G_{2}\right)-\Lambda_{1}\left(G_{1}\right)\right|^{2}+\left|\Lambda_{2}\left(\eta_{2}\right)-\Lambda_{2}\left(\eta_{1}\right)\right|^{2}$.

We show that $\Lambda$ has a unique fixed point.

\section{Lemma 3.}

$$
\Lambda\left(G^{*}, \eta^{*}\right)=\left(G^{*}, \eta^{*}\right)
$$

Proof. Let assume that $\left(G_{i}, \eta_{i}\right)$ are functions in $\mathbb{L}^{2}\left(0 . T ; \mathcal{H} \times V^{\prime}\right)$, and $\left(u_{i}, \varphi_{i}\right)$ are functions obtained in Lemma 1 and Lemma 2 for $(G, \eta)=\left(G_{i}, \eta_{i}\right), i=1,2$. Let $t \in[0 . T]$.

It follows from (1.1) that

$$
\left|G_{2}-G_{1}\right|_{\mathcal{H}}^{2} \leqslant c\left(\left|v_{2}(t)-v_{1}(t)\right|_{V}^{2}+\left|\varphi_{2}(t)-\varphi_{1}(t)\right|_{W}^{2}+\left|u_{2}(t)-u_{1}(t)\right|_{V}^{2}\right) .
$$

Therefore (3.13) yields

$$
\left|G_{2}-G_{1}\right|_{\mathcal{H}}^{2} \leqslant c\left(\left|v_{2}(t)-v_{1}(t)\right|_{V}^{2}+\left|u_{2}(t)-u_{1}(t)\right|_{V}^{2}\right) .
$$

Using (3.5), we find

$$
\begin{aligned}
\left(\dot{v}_{2}(t)-\dot{v}_{1}(t), v_{2}(t)-v_{1}(t)\right)+\left(\mathcal{A} \varepsilon\left(v_{2}(t)\right)-\mathcal{A} \varepsilon\left(v_{1}(t)\right), v_{2}(t)-v_{1}(t)\right)+ & \\
+\left(\eta_{2}(t)-\eta_{1}(t), v_{2}(t)-v_{1}(t)\right)+ & j\left(G_{2}, v_{2}(t)\right)-j\left(G_{2}, v_{1}(t)\right)- \\
& -j\left(G_{1}, v_{2}(t)\right)+j\left(G_{1}, v_{1}(t)\right) \leqslant 0
\end{aligned}
$$

and we have

$$
\begin{aligned}
j\left(G_{2}, v_{2}(t)\right) & -j\left(G_{2}, v_{1}(t)\right)-j\left(G_{1}, v_{2}(t)\right)+j\left(G_{1}, v_{1}(t)\right) \leqslant \int_{\Gamma_{3}} \mu p\left|R G_{2 \nu}\right|\left|v_{2 \tau}\right| d a- \\
& -\int_{\Gamma_{3}} \mu p\left|R G_{2 \nu}\right|\left|v_{1 \tau}\right| d a-\int_{\Gamma_{3}} \mu p\left|R G_{1 \nu}\right|\left|v_{2 \tau}\right| d a+\int_{\Gamma_{3}} \mu p\left|R G_{1 \nu}\right|\left|v_{1 \tau}\right| d a .
\end{aligned}
$$

Moreover, using the property of $R$, we find from (2.11), (2.17) that

$$
j\left(G_{2}, v_{2}(t)\right)-j\left(G_{2}, v_{1}(t)\right)-j\left(G_{1}, v_{2}(t)\right)+j\left(G_{1}, v_{1}(t)\right) \leqslant c\left|G_{2}-G_{1}\right|_{\mathcal{H}}\left|v_{2}-v_{1}\right|_{V} .
$$

Then (3.20) can be rewritten as

$$
\begin{aligned}
\left(\dot{v}_{2}(t)-\dot{v}_{1}(t), v_{2}(t)-v_{1}(t)\right)_{V^{\prime} \times V}+\left(\mathcal{A} \varepsilon\left(v_{2}(t)\right)-\mathcal{A} \varepsilon\left(v_{1}(t)\right), v_{2}(t)-v_{1}(t)\right)+ \\
+\left(\eta_{2}(t)-\eta_{1}(t), v_{2}(t)-v_{1}(t)\right) \leqslant c\left|G_{2}-G_{1}\right|_{\mathcal{H}}\left|v_{2}-v_{1}\right|_{V} .
\end{aligned}
$$

We integrate this inequality with respect to time. We use the initial conditions $v_{1}(0)=$ $=v_{2}(0)=v_{0}$, relation (2.7) and Cauchy-Schwarz' inequality for all $t \in[0, T]$. Then, using the inequality $2 a b \leqslant \frac{a^{2}}{m_{\mathcal{A}}}+m_{\mathcal{A}} b^{2}$, we obtain

$$
\begin{aligned}
& \frac{1}{2}\left|v_{2}(t)-v_{1}(t)\right|_{V}^{2}+\frac{m_{\mathcal{A}}}{2} \int_{0}^{t}\left|v_{2}(s)-v_{1}(s)\right|_{V}^{2} d s \leqslant \frac{1}{2 m_{\mathcal{A}}} \int_{0}^{t}\left|\eta_{2}(s)-\eta_{1}(s)\right|_{V^{\prime}}^{2} d s+ \\
& +\frac{m_{\mathcal{A}}}{2} \int_{0}^{t}\left|v_{2}(s)-v_{1}(s)\right|_{V}^{2} d s+c\left(\int_{0}^{t}\left|G_{2}(s)-G_{1}(s)\right|_{\mathcal{H}}^{2} d s+\int_{0}^{t}\left|v_{2}(s)-v_{1}(s)\right|_{V}^{2} d s\right) .
\end{aligned}
$$


We use Gronwall's inequality to obtain

$$
\left|v_{2}(t)-v_{1}(t)\right|_{V}^{2} \leqslant c\left(\int_{0}^{t}\left|G_{2}(s)-G_{1}(s)\right|_{\mathcal{H}}^{2} d s+\int_{0}^{t}\left|\eta_{2}(s)-\eta_{1}(s)\right|_{V^{\prime}}^{2} d s\right) .
$$

On the other hand

$$
\left|\eta_{2}(t)-\eta_{1}(t)\right|_{V^{\prime}}^{2} \leqslant c\left(\left|\varphi_{2}(t)-\varphi_{1}(t)\right|_{W}^{2}+\left|u_{2}(t)-u_{1}(t)\right|_{V}^{2}\right) .
$$

Therfore (3.13) gives

$$
\left|\eta_{2}(t)-\eta_{1}(t)\right|_{V^{\prime}}^{2} \leqslant c\left(\left|u_{2}(t)-u_{1}(t)\right|_{V}^{2}\right)
$$

Using (3.5), we find

$$
\begin{aligned}
&\left(\dot{v}_{2}(t)-\dot{v}_{1}(t), v_{2}(t)-v_{1}(t)\right)+\left(\mathcal{A} \varepsilon\left(v_{2}(t)\right)-\mathcal{A} \varepsilon\left(v_{1}(t)\right), v_{2}(t)-v_{1}(t)\right)+ \\
&+\left(\eta_{2}(t)-\eta_{1}(t), v_{2}(t)-v_{1}(t)\right)+ j\left(G_{2}, v_{2}(t)\right)-j\left(G_{2}, v_{1}(t)\right)- \\
&-j\left(G_{1}, v_{2}(t)\right)+j\left(G_{1}, v_{1}(t)\right) \leqslant 0 .
\end{aligned}
$$

We integrate this inequality with respect to time. We use the initial conditions $v_{1}(0)=$ $=v_{2}(0)=v_{0}$, relations $(2.7),(3.22)$ and the Cauchy-Schwarz' inequality for all $t \in[0, T]$. Then, using the inequality $a b \leqslant c\left(a^{2}+b^{2}\right)$, we obtain

$$
\int_{0}^{t}\left|v_{2}(s)-v_{1}(s)\right|_{V}^{2} d s \leqslant c\left(\int_{0}^{t}\left|\eta_{2}-\eta_{1}\right|_{V^{\prime}}^{2} d s+\int_{0}^{t}\left|G_{2}-G_{1}\right|_{\mathcal{H}}^{2} d s\right) .
$$

Considering inequalities (3.19) and (3.29), we obtain (3.19) in the following form

$$
\left|G_{2}(t)-G_{1}(t)\right|_{\mathcal{H}}^{2} \leqslant c\left(\int_{0}^{t}\left|\eta_{2}(s)-\eta_{1}(s)\right|_{V^{\prime}}^{2} d s+\int_{0}^{t}\left|G_{2}(s)-G_{1}(s)\right|_{\mathcal{H}}^{2} d s\right) .
$$

We find from (3.9), (3.27) and (3.29) that

$$
\left|\eta_{2}-\eta_{1}\right|_{V^{\prime}}^{2} \leqslant c\left(\int_{0}^{t}\left|\eta_{2}-\eta_{1}\right|_{V^{\prime}}^{2} d s+\int_{0}^{t}\left|G_{2}-G_{1}\right|_{\mathcal{H}}^{2} d s\right) .
$$

Using (3.16), we obtain that

$$
\left|\Lambda\left(G_{2}, \eta_{2}\right)-\Lambda\left(G_{1}, \eta_{1}\right)\right|^{2} \leqslant c \int_{0}^{t}\left|\left(G_{2}, \eta_{2}\right)-\left(G_{1}, \eta_{1}\right)\right|_{\mathcal{H} \times V^{\prime}}^{2} d s .
$$

It follows from (3.32) by recurrence that

$$
\left|\Lambda^{p}\left(G_{2}, \eta_{2}\right)-\Lambda^{p}\left(G_{1}, \eta_{1}\right)\right|_{\mathbb{L}^{2}\left(0 . T ; \mathcal{H} \times V^{\prime}\right)}^{2} \leqslant \frac{(c t)^{p}}{p !}\left|\left(G_{2}, \eta_{2}\right)-\left(G_{1}, \eta_{1}\right)\right|_{\mathbb{L}^{2}\left(0 . T ; \mathcal{H} \times V^{\prime}\right)}^{2} .
$$

This inequality shows that for a sufficiently large $p$ the operator $\Lambda^{p}$ is a contraction operator in the Banach space $\mathbb{L}^{2}\left(0, T \mathcal{H} \times V^{\prime}\right)$. Therefor, there exists a unique element $\left(G^{*}, \eta^{*}\right) \in$ $\mathbb{L}^{2}\left(0, T \mathcal{H} \times V^{\prime}\right)$ such that

$$
\Lambda\left(G^{*}, \eta^{*}\right)=\left(G^{*}, \eta^{*}\right) .
$$

We find from (3.16) that

$$
\left(G^{*}, \eta^{*}\right)=\left(\sigma_{G^{*} \eta^{*}}, \xi^{*} \nabla \varphi_{G^{*} \eta^{*}}+\mathcal{G} \varepsilon\left(u_{G^{*} \eta^{*}}\right)\right) .
$$


Now we have everything that is required to prove Theorem 1.

Proof of Theorem 1. Existence. Let $\left(G^{*}, \eta^{*}\right) \in \mathbb{L}^{2}\left(0, T \mathcal{H} \times V^{\prime}\right)$ be the fixed point of $P V_{G \eta}$. Let $\left(u^{*}, \varphi^{*}\right)$ be the solution to Problems $P V_{G \eta}$ and $P V 1_{G \eta}$ for $(G, \eta)=\left(G^{*}, \eta^{*}\right)$, that is, $u^{*}=u_{G^{*} \eta^{*}}$ and $\varphi^{*}=\varphi_{G^{*} \eta^{*}}$. It results from $(2.20)$ and $(2.21)$ that $\left(u^{*}, \varphi^{*}\right)$ is a solution of Problem $P V$. Properties (3.1) and (3.2) follow from Lemma 1 and Lemma 2.

Uniqueness. The uniqueness of the solution is a consequence of the uniqueness of the fixed point of operator (3.16).

\section{References}

[1] V.Barbu, Optimal Control of Variational Inequalities, Pitman, Boston, 1984.

[2] R.C.Batra, J.S.Yang, Saint Venant's principle in linear piezoelectricity, Journal of Elasticity, 38(1995), 209-218.

[3] M.Barboteu, J.R.Fernández, Y.Ouafik, Numerical analysis of a frictionless viscoelastic piezoelectric contact problem, M2AN Math. Model. Numer. Anal., (in press).

[4] H. Brézis, Analyse Fonctionnelle, Théorie et Application, Masson, Paris, 1987.

[5] O.Chau, On a class of second order evolution inéquality and application, Int. J. of Appl. Math. and Mech., 4(2008), no. ,1 24-48.

[6] G.Duvaut, J.L.Lions, Inequalities in Mechanics and Physics, Springer-Verlag, Berlin, 1988.

[7] W.Han, M.Sofonea, K.Kazmi, Analysis and numerical solution of a frictionless contact problem for electro-elastic-visco-plastic materials, Comput. Methods Appl. Mech. Engrg., 196(2007), 3915-3926.

[8] J.L.Lions, Quelques Méthodes de Résolution des Problémes Aux Limites non Linéaires, Dunod et Gauthier-Villars, Paris 1969.

[9] R.D.Mindlin, Polarisation gradient in elastic dielectrics, Int. J. Solids Structures, 4(1968), 637-663.

[10] R.D.Mindlin, Continuum and lattice theories of influence of electromechanical coupling on capaci- tance of thin dielectric films, Int. J. Solids, 4(1969), 1197-1213.

[11] R.D.Mindlin, Elasticity, Piezoelectricity and Cristal lattice dynamics, J. of Elasticity, 4(1972), 217-280.

[12] M.Rochdi, M.Shillor, M.Sofonea, A quasistatic contact problem with directional friction and damped response, Applicable Analysis, 68(1998), 409-422.

[13] L.Selmani, N.Bensebaa, An Electro-viscoelastic Contact Problem with Adhesion and Damage, Rend. Sem. Mat. Univ. Pol. Torino, 66(2008), no. 2, 151-171.

[14] M.Sofonea, E.-H.Essoufi, Quasistatic frictional contact of a viscoelastic piezoelectric body, Adv. Math. Sci. Appl., 14(2004), no. 1, 25-40.

[15] M.Selmani, L.Selmani, Frictional contact problem for elastic-viscoplastic materials with thermal effect, Berlin Heidelberg, 2013.

[16] M.Sofonea, M.Shillor, Variational analysis of quasistatic viscoplastic contact problems with friction, Communications in Applied Analysis, 5(2001), 135-151. 


\title{
Вариационный анализ динамической электро-вязкоупругой задачи с трением
}

\author{
Азиза Бачмар \\ Сурая Бутечебак \\ Туффик Серрар \\ Алжир
}

Кафедра математики, факультет наук

Ферхат Аббас Университет Сетиф-1, 19000

\begin{abstract}
Изучается проблема динамического контакта. Поведение материала моделируется пъезоэлектрическими эффектами для электровязкоупругого конститутивного закона. Тело может вступить в контакт с жестким препятствием. Контакт описывается условием Синъорини, разновидностью закона сухого трения Кулона и условием регуляризованной электрической проводимости. Мы выводим вариационную постановку задачи, а затем, в предположении малости коэфбициента трения, доказываем существование и результат единственности слабого решения для модели. Доказательство основано на аргументах эволюиионных вариачионных неравенств и неподвижных точек операторов.
\end{abstract}

Ключевые слова: пъезоэлектрический, фрикционный контакт, вязкоупругий, неподвижная точка, динамический процесс, закон трения Кулона, вариационный неравенство. 